\title{
MANEJO ESTOMATOLÓGICO DE LA HIPOMINERALIZACIÓN INCISIVO MOLAR. REPORTE DE CASO
}

\section{MANAGEMENT OF PATIENT WITH MOLAR INCISOR HYPOMINERALISATION. A CASE REPORT}

\author{
Romo-Cardoso Adriana ${ }^{1 *}$, Moncayo-Pinos Jaime ${ }^{2}$, Aguilar Jonnathan ${ }^{3}$ \\ ${ }^{1}$ CD. Esp. -Docente de la Universidad Católica de Cuenca. Ecuador \\ ${ }^{2}$ Estudiante de la Universidad Católica de Cuenca ${ }^{3}$ Estudiante de la Universidad Católica de Cuenca. Ecuador \\ *aromoc@ucacue.edu.ec
}

\begin{abstract}
Resumen
La hipomineralización incisivo molar (HIM), es un defecto estructural del esmalte de etiología no específica, esta enfermedad presenta una prevalencia que oscila entre el 12-19\% en Latino América. OBJETIVO: realizar una revisión de literatura respecto a las características clínicas asociadas con la HIM, su manejo odontológico y opciones de tratamiento restaurador en un paciente de 8 años 11 meses; que asistió a la Clínica de Estudios de Posgrado, de Odontología Pediátrica de la Facultad de Estomatología Cayetano Heredia, Lima - Perú. Como conclusiones se prestó especial atención al tratamiento preventivo del niño ante la hipersensibilidad severa, el riesgo cariogénico y factor estético. El tratamiento fue exitoso el cual junto con un asesoramiento dietético y fisioterapia oral mejoró la calidad de vida del paciente.
\end{abstract}

Palabras clave: Hipomineralización incisivo-molar, esmalte dental, caries dental, terapia preventiva, asesoramiento dietético, técnicas de higiene.

\begin{abstract}
Incisor-Molar hypomineralization $(M I H)$ is a structural defect of the enamel with non-specific etiology; this disease presents a prevalence that oscillates between 12-19\% in Latin America. AIM review of the literature regarding the clinical characteristics associated with $\mathrm{MIH}$, dental management and restorative treatment options. We present a case of 8 years and 11 months old patient treated at the graduate clinic of Facultad de Odontología Pediátrica de la Facultad de Estomatología Cayetano Heredia, Lima - Perú. Conclusion: special attention was given to the preventive treatment of the child in case of severe hypersensitivity, cariogenic risk and aesthetic consideration. The treatment was successful, supported with dietary advice and oral physiotherapy improved the quality of life of the patient.
\end{abstract}

Key words: Incisor-molar hipomineralization, dental enamel, dental caries, preventive therapy, dietary advice, hygiene techniques.

\section{INTRODUCCIÓN}

La terminología HIM sugerida en el año 2001 por Weerheijm y cols. ${ }^{1}$ describe una condición clínica caracterizada por defectos de hipomineralización del esmalte que afectan desde uno a cuatro de los primeros molares y que está frecuentemente asociada a opacidades en los incisivos y molares. ${ }^{1}$ Este defecto favorece a la acumulación de placa bacteriana y sumado a la falta de cepillado por la hipersensibilidad dental del paciente conlleva fácilmente a un riesgo estomatológico alto. ${ }^{1}$ Las anomalías estructurales de los dientes se asocian con alteraciones producidas durante la odontogénesis y su origen puede vincularse a factores locales o sistémicos. El ameloblasto, productor del esmalte dental, célula que es sensible a cambios, incluso menores, que se reflejarán en defectos a modo de cicatrices clínicamente perceptibles o cambios severos, esto puede conducir a su muerte y alteraciones clínicas importantes. De acuerdo con la época de aparición, estos factores afectarán a la dentición decidua, permanente o ambas. Histológicamente, existe una conservación de la microestructura del ameloblasto, la cual funciona de manera normal durante la fase secretora. Sin embargo, existe una menor compactación y organización 
de los cristales en áreas porosas, ocasionada en la fase de maduración, es decir el problema sucede durante el último período prenatal y los primeros años de vida. ${ }^{2,9}$

En relación a la prevalencia estudios realizados en Argentina, Chile, Uruguay y Brasil reportan que oscila entre $12 \%$ al $40,2 \%$, siendo esta mas frecuente en el sexo masculino según el estudio de López. ${ }^{3}$ Aun la etilogia resulta poco clara, varios autores señalan causas sistémicas para su origen y entre los factores relacionados con la madre se encuentran, parto prematuro, cianosis, anemia, tabaquismo, cesárea, el uso frecuente de antibióticos y enfermedad celíaca. Entre los causales relacionados con el niño se encuentran enfermedades de la primera infancia como varicela, otitis, infecciones urinarias, amigdalitis, fiebre alta. ${ }^{4,5,7,14}$ La relevancia de cada evento es difícil establecer ya que muchos de estos pueden suceder más de una vez durante la primera infancia. ${ }^{10}$ Clínicamente la HIM se manifiesta en el esmalte de 3 formas: opacidades anormales y delimitadas sin descomposición de la superficie del esmalte que presentan una coloración blanquecino cremoso (media), marrón amarillento (moderada) y una descomposición pos-eruptiva del esmalte (severa). ${ }^{10,11}$ Se debe destacar que no todos los pacientes con HIM presentan opacidades en el esmalte de sus incisivos permanentes, además el grado de hipomineralización variará en los dientes de un mismo individuo, los dientes que presentan mayor afectación son los incisivos centrales superiores. ${ }^{2,7} \mathrm{La}$ patología causa serios problemas para los niños afectados y sus familia a consecuencia del dolor, destrucción, compromiso funcional, estético, y dificultad de tratamiento; por este motivo la preocupación por la entidad va en aumento., ${ }^{1,5,12}$ Dependiendo de la severidad del caso, edad del agente etiológico, edad del presentación del paciente en la clínica dental y la sensibilidad a estímulos de aire y mecánicos; el paciente puede presentar incapacidad para masticar alimentos, caries dental y sus secuelas. En condiciones severas puede estar presente la incapacidad para cepillar los dientes. ${ }^{13}$ Los dientes tratados pueden ser muy sensibles, es por esto que se recomienda no dirigir aire frio al momento de la exploración. La sensibilidad puede estar asociada a: Perdida pos-eruptiva del esmalte, sensibilidad por inflamación pulpar subyacente sensibilidad extrema. ${ }^{14}$ El tratamiento de sensibilidad puede lograrse con: restauración de tejido perdido, aplicación de barniz fluorado y uso diario de fosfopéptido de caseína con fosfato de calcio amorfo (mousse dental). No se debe usar si existe alergia a productos lácteos. ${ }^{14} \mathrm{~A}$ consecuencia de la sensibilidad presente en el momento del manejo del paciente Dugal y cols ${ }^{14}$ recomiendan usar articaína en lugar de lidocaína, complementar con analgesia intra-ligamentosa y de ser necesario usar sedantes inhalatorios para disminuir la ansiedad, aislamiento absoluto con dique de goma evitando la sensibilidad de molares no sensibles y no usar aspiración de volumen alto. ${ }^{1-4}$ En el tratamiento preventivo es importante empezar a tratar a los niños afectados y sus padres con el asesoramiento dietético. El fosfato de calcio fosofopéptido amorfo (CPP-ACP) se ha incorporado en la goma de mascar sin azúcar y promueve a la remineralización de las lesiones de caries debajo de la superficie . A raíz de estos hallazgos, se ha sugerido empíricamente que la aplicación de una pasta dental que contiene CPP-ACP ayudará a sellar, desensibilizar y actuar como una fuente de calcio y fosfato biodisponible. ${ }^{10}$ La opacidad en la superficie vestibular de los incisivos puede molestar al paciente. Las lesiones superficiales se pueden eliminar con micro-abrasión, de ser profunda y a pesar de que existen complicaciones, los siguientes pasos darán buen resultado: Fresas de alta velocidad para eliminar alrededor de la mitad de la superficie en donde esta la opacidad, colocar un tono opaco como primera capa enmascarando la lesión y cobertura del espesor del esmalte con tonos de resina apropiados. $^{15}$

\section{REPORTE DE CASO}

Paciente de sexo masculino de 8 años y 11 meses de edad, procedente de la ciudad de Lima - Perú. Acude a la clínica del posgrado de Odontopediatría de la Universidad Peruana Cayetano Heredia, sin presentar ningún riesgo sistémico, la madre refiere "Mi hijo tiene sensibilidad en sus muelitas y cambio de color" y visitó por última vez al dentista una semana atrás. A la evaluación del cuestionario revela que el niño cepilla los dientes $2-3$ veces al día, es alérgico al polvo y a la pelusa, presentó complicaciones en el parto (hipoxia al nacer), el niño fue alimentado con leche materna hasta el año y medio y considera que la salud familiar oral era mala. Al examen clínico general revela una apreciación física normal y sana, al examen clínico estomatológico presenta onicofagia, paladar profundo, lengua saburral, retraso en la cronología y secuencia de erupción, múltiples lesiones cariosas cavitadas, y pérdida de estructura dentaria no asociada a caries en las piezas 16, 26, 36, 46, 31, 41 y 42; riesgo estomatológico alto.

\section{PLAN DE TRATAMIENTO}

1. Examen clínico estomatológico.

2. Set fotográfico.

3. Análisis dietético.

4. Set radiográfico.

\section{ANÁLISIS DIETÉTICO}

Se hablo con la madre la cual menciona que el niño en casa consume 5 comidas diarias de estas 4 son preparadas en casa y la restante en la escuela la cual incluye: alimentos de azúcar procesada y frutas además la madre agrego que a los líquidos que ingiere su hijo le agrega una cucharada y media de azúcar. Esto hace referencia a que el niño tiene aproximadamente 6 golpes de azúcares diarios.

\section{ANÁLISIS RADIOGRAFÍCO}

- Pieza. 1.6: Imagen radiolúcida (IRL) lineal en cara libre compatible con surco profundo. 


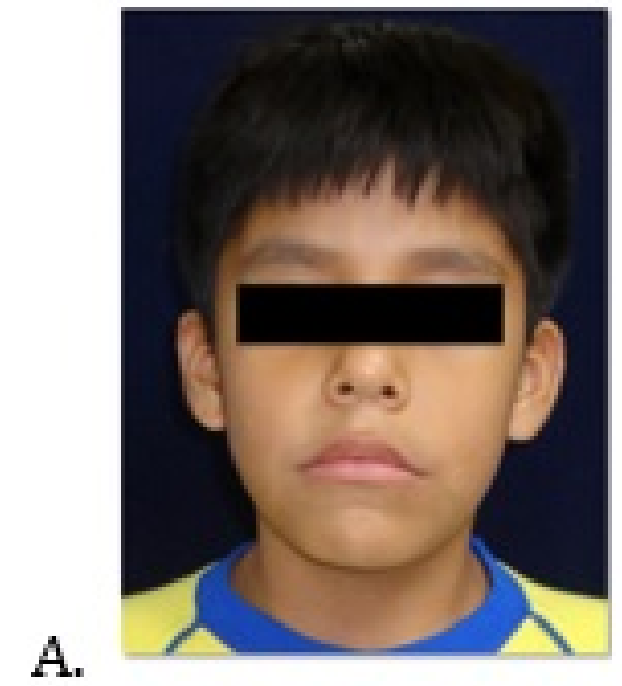

A.

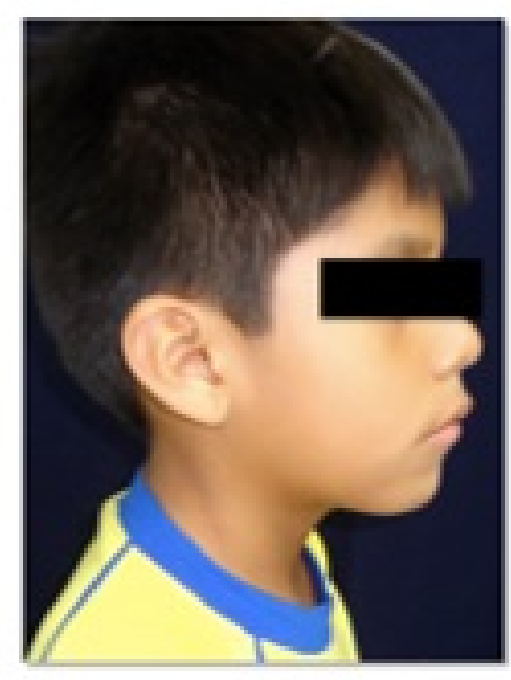

B.

Fig. 1. A. Foto de frente: Dolicofacial, Facies no característica B. Foto de Perfil: perfil convexo, labios competentes

- Pieza 5.5: IRL ocluso-mesial compatible con lesión cariosa, compromiso de esmalte y dentina (R2).

- Pieza 2.6: Alteración de la morfología coronaria caracterizada por la ausencia de ángulos cuspídeos.

- Pieza 6.5: IRL mesial compatible con lesión cariosa, compromiso esmalte dentina cerca de cámara pulpar (R3).

- Pieza 6.4: IRL distal compatible con lesión cariosa R3.

- Pieza 3.6: IRL lineal proyectada en cara libre compatible con surco profundo.

- Pieza 7.5: Restauración radiopaca coronaria oclusal con lesión de caries recidivante.

- Pieza 7.4: IRL oclusal compatible con lesión cariosa R3.

- Pieza 4.6: Defecto en esmalte en la cúspide Mesial.
A.

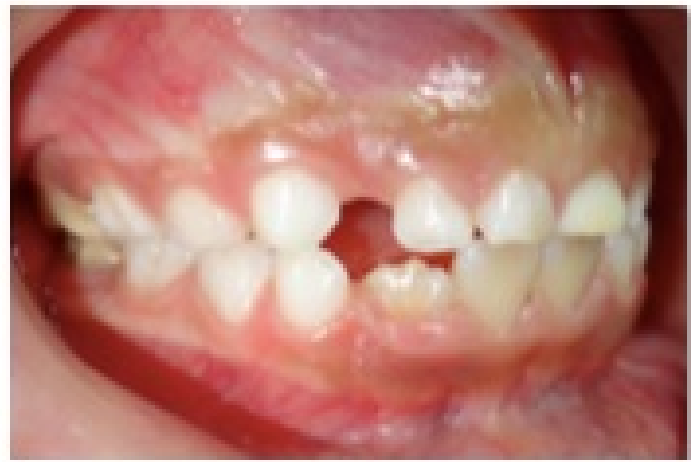

B.

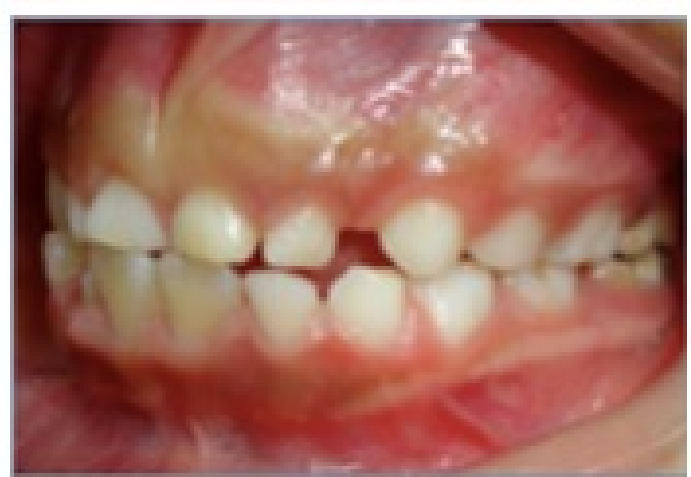

Fig. 2. A. Relación Molar Clase I, Relación Canino Clase II B. Relación Molar Clase I, Relación canina Clase I.

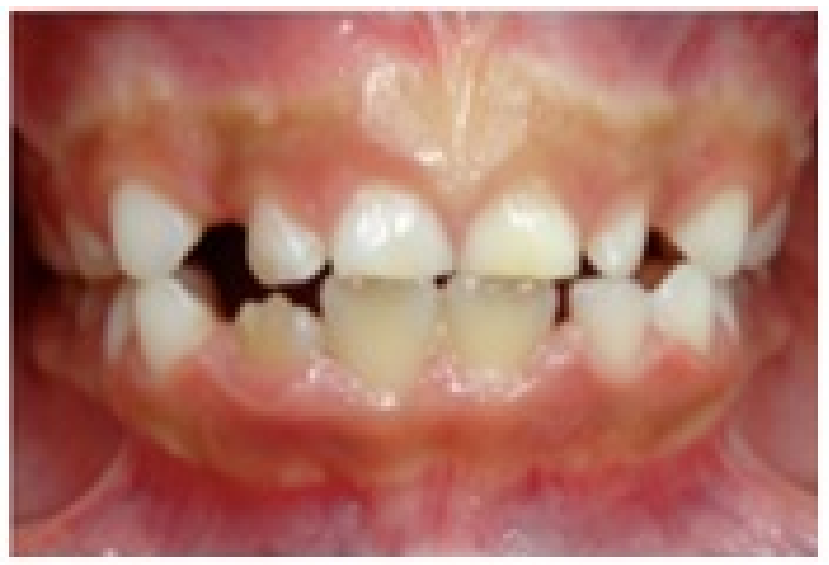

Fig. 3. Opacidades Blanquecino/Cremoso en piezas 3.1, 3.2, 4.1 y 4.2 
A.

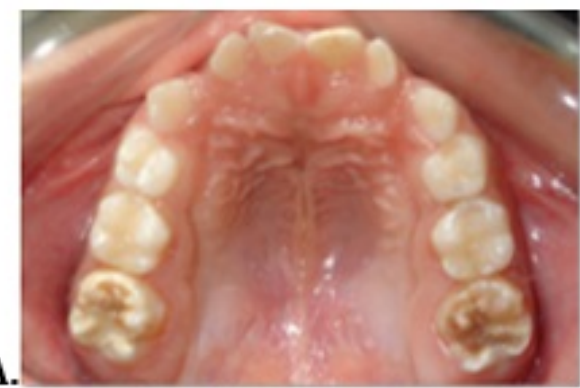

B

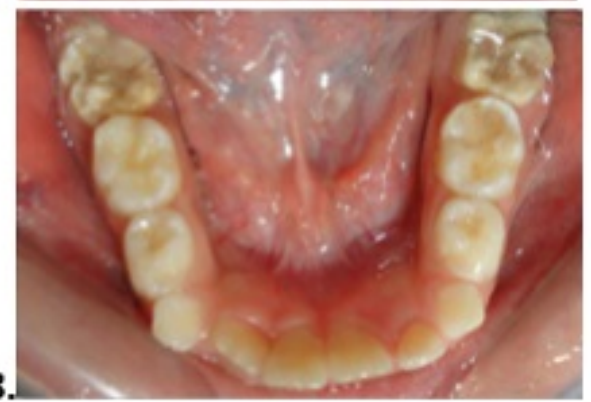

Fig. 4. Superficie oclusal superior e inferior y cambio de coloración y pérdida de estructura dentaria no asociada a caries en las piezas $1.6,2.6,3.6$ y 4 .

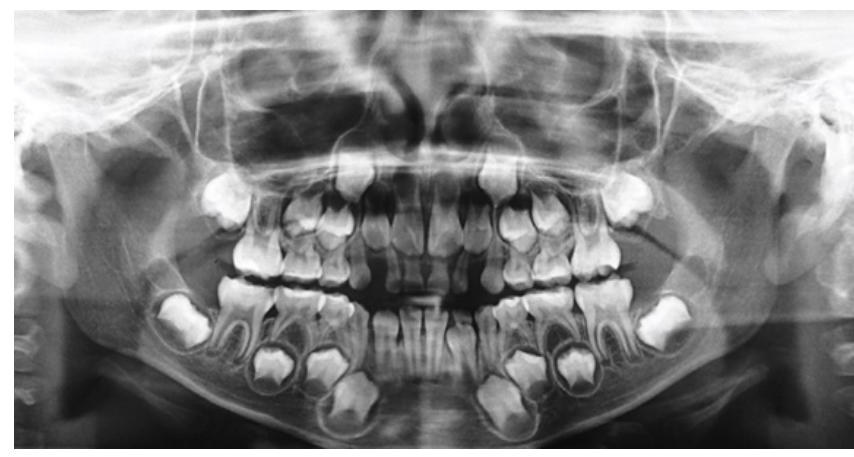

Fig. 5. Radiografía Panorámica: Obsérvese menor radiopacidad en las piezas 2.6, 2.6, 3.6 y 4.6

\section{TRATAMIENTO}

Los Objetivos fueron:

1. Sensibilizar a los padres y al niño sobre su estado de salud bucal.

2. Incentivar la adquisición y práctica de hábitos de higiene bucal.

3. Eliminar y restaurar lesiones cariosas.

4. Prevenir futuras lesiones cariosas.

5. Devolver la estética, fonética y función masticatoria.

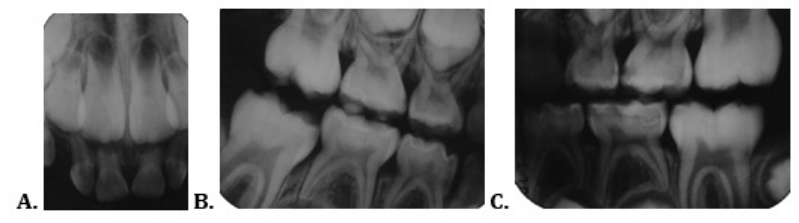

Fig. 6. Periapical de incisivos y bite-wing de molares derecho (B) e izquierdo (C).

6. Revertir la hipersensibilidad de las piezas con HIM.

7. Corregir la mal-oclusión.

8. Disminuir el riesgo estomatológico.

Se realizó una profilaxis y aplicación de barniz fluorado (Fluoruro de Sodio al 5\%) en todas las piezas dentales realizada la primera fase de prevención, la fase restauradora mediante una técnica la restauración con resina en las piezas dentales: 5.5 ocluso-mesial, 6.5 ocluso-mesial, 6.4 oclusodistal, 7.4 oclusal; 7.5 ocluso-mesial; 8.4 oclusal; 8.5 oclusal; aplicación de sellante en pieza 5.4; tratamiento restaurador atraumático (TRA) que según Otazú22 "es la remoción del tejido dental infectado empleando instrumentos manuales" son presentados como métodos efectivo evitando la perdida de estructura sana, para la posterior restauración con materiales adhesivos (ionómero de vidrio) en piezas 16, 26, 36 y 46. Al finalizar la fase de mantenimiento consistió en evaluar las piezas con HIM las mismas que se restauraron con ionómero de vidrio y se considera en un futuro posibles restauraciones con incrustaciones o coronas a partir de los 16 años de edad (transferencia al servicio de Rehabilitación Oral).

\section{DISCUSIÓN}

La HIM es un defecto estructural del esmalte asociada a defectos hereditarios, locales y sistémicos que afecta a incisivos y molares deciduos, permanentes o ambos dependiendo de la época de aparición del agente causal, la condición clínica afecta desde 1 a 4 molares y esta frecuentemente asociada a opacidades en incisivos, pudiendo variar el grado del defecto en distintas piezas dentarias de un mismo paciente. ${ }^{1-3,7}$ La prevalencia reportada por diferentes autores varia, de un $2,4 \%$ a un $44 \%$ en el mundo. ${ }^{1,16-20}$ En la población latinoamericana varios estudios fueron realizados encontrándose en Argentina un 16,1 \% ${ }^{2}$, del 12,3\% en Uruguay, ${ }^{4} 16,8 \%$ en Chile, ${ }^{21}$ y en Brasil el $40,2 \%{ }^{6}$ Se han propuesto varias causas del origen de la HIM, ya sea por contaminantes ambientales, problemas pre o post natales, enfermedades comunes de la infancia o exposición a sustancias médicas o tóxicas. ${ }^{14}$ La HIM conlleva varios problemas para el infante y su familia, dependiendo de el grado de afectación de las piezas puede llegar a comprometer 
A.

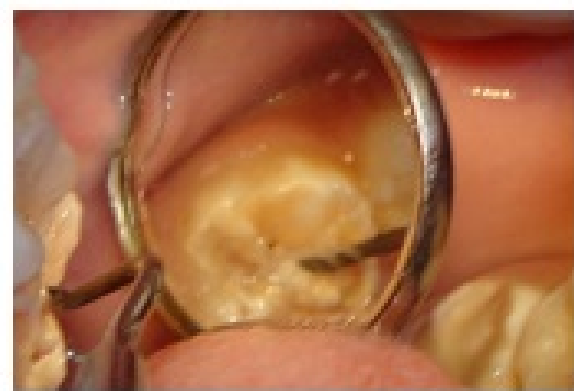

B.

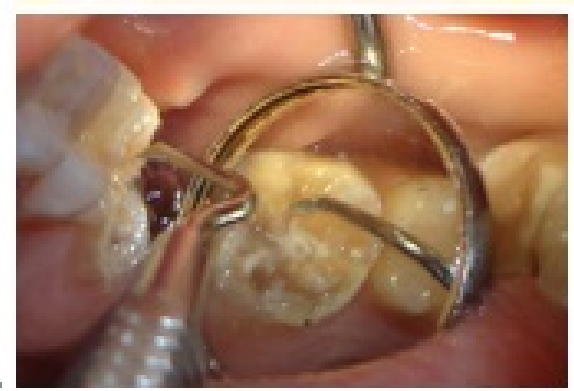

A.

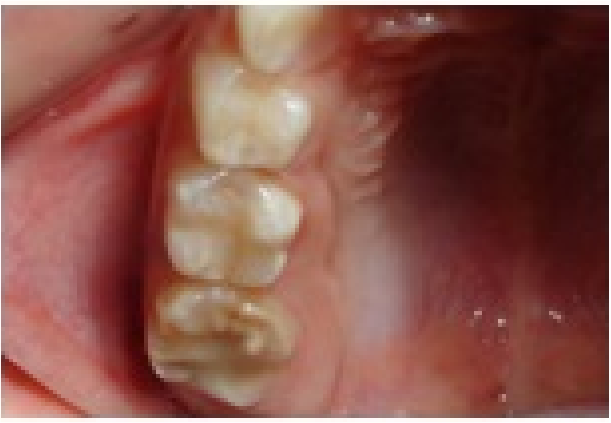

B.

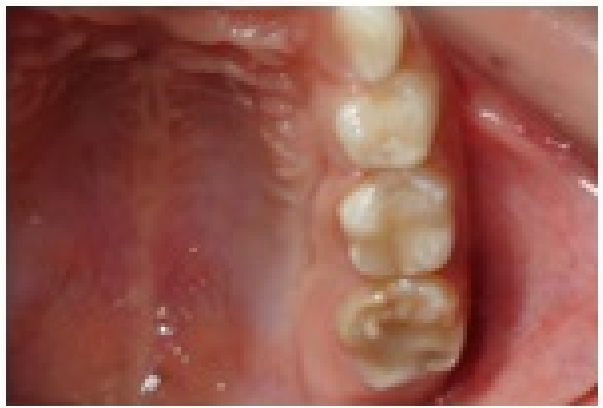

Fig. 7. Remoción de lesión cariosa en piezas afectadas

desde la higiene hasta la alimentación del niño, es por esto que un diagnóstico temprano es importante. ${ }^{13,14}$ Debemos decir que es realmente esencial la preocupación de los padres por la salud oral de su hijo. Las primeras manifestaciones estarán presentes al momento de la erupción del primer molar, es por esta razón que se recomienda la vigilancia del niño. Es fundamental el conocimiento de los padres de las complicaciones que ocasiona la HIM y su relación con la caries dental. El esmalte dental pierde su característica lisa y se torna rugosa e irregular ofreciendo mayor retención de bio-película y dificultando la correcta higiene de la pieza afectada, por lo tanto esto promoverá el desarrollo de lesiones de cariosas. No se debe olvidar que el paciente con HIM requiere una atención especializada, debido a la sensibilidad dental que podría estar presente, las dificultades en cuanto al manejo del paciente. El conocimiento sobre su etiopatogenia será fundamental para el desarrollo de labores con el fin de promocionar una buena salud oral. El desarrollo de un programa de atención con énfasis en la prevención y restauración junto con la vigilancia, son sumamente importante para tratar de mantener los dientes afectados en la cavidad bucal de los niños afectados. Es importante manejar los objetivos del tratamiento cumpliendo los siguientes criterios:

- Orientar a los padres y al niño sobre su estado de salud bucal.

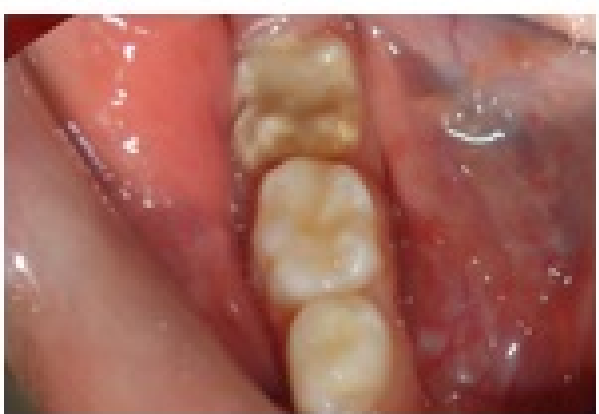

C.

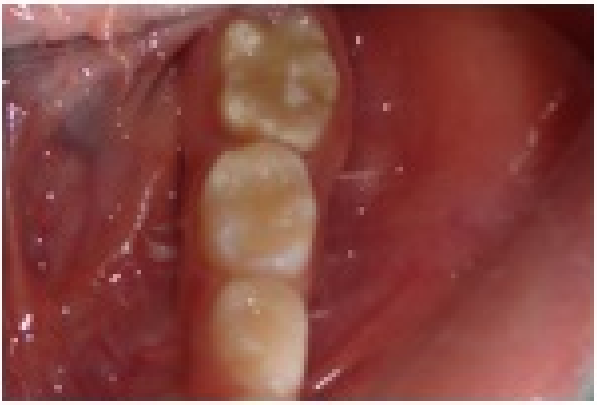

Fig. 8. Aplicación de restauración definitiva con ionómero de vidrio 
- Incentivar la adquisición y práctica de hábitos de higiene bucal.

- Eliminar y restaurar lesiones cariosas.

- Prevenir futuras lesiones cariosas.

- Devolver la estética, fonética y función masticatoria.

- Revertir la hipersensibilidad de las piezas con HIM.

- Corregir la maloclusión.

- Disminuir el riesgo estomatológico.

- Mantener una buena higiene oral.

\section{CONCLUSIONES}

De acuerdo a la literatura estudiada recientemente la prevalencia de HIM parece ir en aumento. Para el éxito clínico es importante el diagnóstico temprano, por lo general los pacientes con HIM son de difícil manejo de conducta por la hipersensibilidad que presenta. La literatura no refiere el material restaurador ideal para incisivos y molares con HIM, depende de la severidad de hipomineralización y de las condiciones del paciente.

Conflicto de intereses y financiamiento Los autores declaran no tener conflicto de intereses, haber cumplido con los requisitos de autoría y haber autofinanciado este artículo.

\section{Referencias}

1 Weerheijm KL, Jalevik B, Alaluusua S. Molarincisorhypomineralization. Caries Res 2001;35:390-391.

2 López Jordi Mc, cortese SG, Álvarez 1, Salveraglio i, Ortolani AM, Biondi AM. comparación de la prevalencia de hipomineralización molar incisiva en niños con diferente cobertura asistencial en las ciudades de Buenos Aires (Argentina) y Montevideo (Uruguay). Salud colectiva.2014;10(2):243251.

3 Farah RA, Monk BC, Swain MV, Drummond BK. Protein content of molar-incisor hypomineralisation enamel. Journal of Dentistry. 2010;38(7):591-596.

4 López Jordi M. del Carmen, Álvarez Licet, Salveraglio Inés. Prevalencia de la Hipomineralización Molar-Incisiva (MIH) en niños con diferente cobertura asistencial (privada y pública) en Montevideo, Uruguay. Odontoestomatología [Internet]. 2013 Nov; 15( 22 ): 4-15. Documento disponible

5 Jalevik B, Noren JG, Barregard L. Etiologic factors inflencing the prevalence of demarcated opacities in permanent first molars in a group of Swedish children. Eur J Oral Sci 2001;109:230-234.

6 Costa-Silva CM, Jeremias F, de Souza JF, Cordeiro RC, Santos-Pinto L, Zuanon AC. Molar incisor hypomineralization: prevalence, severity and clinical consequences in Brazilian children. International Journal of Paediatric Dentistry. 2010;20(6):426-434

7 Soviero V, Haubek D, Trindade C, Da Matta T, Poulsen S. Prevalence and distribution of demarcated opacities and their sequelae in permanent 1 st molars and incisors in 7 to 13-year-old Brazilian children. Acta Odontologica Scandinavica. 2009;67(3):170-175.

8 Whitford GM, Angmar-Månsson B. Fluorosis-like effects of acidosis, but not $\mathrm{NH}+4$, on rat incisor enamel. Caries Research.1995;29(1):20-25.

9 Sui W, Boyd C, Wright JT. Altered $\mathrm{pH}$ regulation during enamel development in the cystic fibrosis mouse incisor. Journal of Dental Research. 2003;82(5):388-392.

10 Souza J.F. et al. Molar Incisor Hypomineralisation: Possible aetiological factors in children from urban and rural areas. European Archives of Paediatric Dentistry 13. 2012

11 Fitzpatrick L. O'Conell A. First permanent molars with molar incisor Hypomineralisation. Journal of the Irish Dental Association Volume 53: 32 - 37, 2007

12 Lygidakis N.A. Treatment modalities in children with teeth affected by molar-incisor enamel hypomineralisation (MIH): A systematic review. European Archives of Paediatric Dentistry // 11 (Issue 2). 2010'

13 Rao MH, Aluru SC, Jayam C, Bandlapalli A, Patel N. Molar Incisor Hypomineralization. J Contempt Dent Pract 2016;17(7):609:613 disponible en: Documento disponible

14 Dugal M., Cameron A., Toumba J. Odontologia pediátrica. Manual moderno, Primera edición. 2014

15 Messer LB. Getting the fluoride balance right: Children in long-term fluoridated communities. Synopses 2005;30:710.

16 Ahmadi R. Ramazani N. y Col. MIH: A Study of Prevalence and Etiology in a Group of Iranian Children. Iranian Journal of Pediatrics, Volume 22, June 2012, Pages: 245-251

17 Souza J.F. et al. Molar Incisor Hypomineralisation: Possible aetiological factors in children from urban and rural areas. European Archives of Paediatric Dentistry 13. 2012

18 Messer LB. Getting the fluoride balance right: Children in long-term fluoridated communities. Synopses 2005;30:7-10

19 Comes Martínez A, Puente Ruiz C, Rodríguez Salvanes F. Prevalencia de hipomineralización en primeros molares permanentes $(\mathrm{MIH})$ en población infantil del Área 2 de Madrid. RCOE [Internet]. 2007;12(3):129-134. disponible en: Documento disponible

20 Mahoney EK, Moorinson DG. The prevalence of MolarIncisor Hypomineralisation (MIH) in Wainuiomata children. The New Zealand Dental Journal. 2009;105(4):121127.

21 Jans, M. A.; Díaz, M. J.; Vergara, G. C. \& Zaror, S. C. Frecuencia y severidad de la hipomineralización molar incisal en pacientes atendidos en las clínicas odontológicas de la Universidad de La Frontera. Int. J. Odontostomat. 2011; 15(2):133-140

Recibido: 25 de Julio de 2016

Aceptado: 10 de Agosto de 2016 